Special issue of the International Conference on Computational and Experimental Science and Engineering (ICCESEN 2014)

\title{
Study of a Mixed Hadronic-QGP System with Massive Particles
}

\author{
L. Ghenam* And A.A. El Djoudi \\ Laboratoire de Physique des Particules et Physique Statistique, Ecole Normale Supérieure, \\ Kouba Physics Department, BP 92 16050, Vieux-Kouba, Algiers, Algeria
}

\begin{abstract}
We consider a mixed system of hadronic and quark-gluon plasma (QGP) phases in a finite volume, and use a phenomenological approach with two phases matter equations of state to study the occurring deconfinement phase transition with varying temperature and/or chemical potential. We take into account massive particles in both phases, and calculate several physical quantities describing well the system at the phase transition. We probe the behaviour of these quantities, by examining their variations with temperature and volume at fixed chemical potential, as well as with chemical potential and volume at fixed temperature, in three dimensional plots, and obtain the finite size effects on the deconfinement phase transition.
\end{abstract}

DOI: 10.12693/APhysPolA.128.B-341

PACS: 12.38.Mh, 12.39.Ba, 05.70.--a, 05.70.Ce, 05.70.Fh, 64.10.+h

\section{Introduction}

It is well confirmed that quantum chromodynamics (QCD) at finite temperature exhibits a behaviour of a phase transition, since at sufficiently high temperatures and/or densities, quarks and gluons are no more confined into hadrons. Strongly interacting matter undergoes a phase transition from hadronic state to a deconfined state called "quark-gluon plasma" (QGP), which seems to have existed in the early moments of the universe, up to times $\approx 10^{-5} \mathrm{~s}$, and which could be created in ultrarelativistic heavy-ion collisions occurring in accelerators. There, collisions take place in a finite size, and the study of the effects of size finitude is necessary. Phase transitions are known to be infinitely sharp, signaled by some singularities, only in the thermodynamic limit [1]. In previous works $[2,3]$, one of us has studied such effects for the deconfinement phase transition (DPT) using massless particles in a first approach. In the present work, we take into account massive particles in both phases, and calculate several physical quantities allowing to probe the behaviour of the system at the phase transition as in $[4,5]$, but now we examine the finite size effects for the thermal (temperature driven) DPT as well as for the density driven DPT. We use the phase coexistence model [2,3], and calculate the equations of states of both hadronic gas phase (H-phase) constituted of massive pions and QGP phase containing massive strange quarks additionally to massless $u$ and $d$ quarks. The mixed H-QGP phase system has a finite volume: $V=V_{H}+V_{Q G P}$, and the parameter $h$ representing the fraction of volume occupied by the H-phase: $V_{H}=h V$ is then defined. Thus, the value $h=1$ corresponds to a total $\mathrm{H}$-phase and $h=0$ to a total QGP phase.

\section{Finite size rounding for the thermal and density driven deconfinement phase transition}

We probe the behaviour of the most important thermodynamical quantities by examining their variations with temperature $T$ and volume $V$ at vanishing chemical potential $\mu=0$, as well as with chemical potential and volume at fixed temperature, using the common value $B^{1 / 4}=145 \mathrm{MeV}$ for the bag constant. The main quantities of interest are the order parameter $\langle h(T, V, \mu)\rangle$, which is simply in this case the mean value of the hadronic volume fraction, the entropy density $\langle s(T, V, \mu)\rangle$ and the energy density $\langle\varepsilon(T, V, \mu)\rangle$, where:

$$
\begin{aligned}
& \langle s(T, V, \mu)\rangle=s_{Q G P}+\left(s_{H}-s_{Q G P}\right)\langle h(T, V, \mu)\rangle \\
& \{\varepsilon(T, V, \mu)\rangle=\varepsilon_{Q G P}+\left(\varepsilon_{H}-\varepsilon_{Q G P}\right)\langle h(T, V, \mu)\rangle
\end{aligned}
$$

with:

$$
\begin{aligned}
& \langle h(T, V, \mu)\rangle=\frac{-\frac{V}{T}\left(f_{H}-f_{Q G P}\right)-1}{-\frac{V}{T}\left(f_{H}-f_{Q G P}\right)} \\
& \times \frac{\exp \left(-\frac{V}{T}\left(f_{H}-f_{Q G P}\right)\right]+1}{\exp \left(-\frac{V}{T}\left(f_{H}-f_{Q G P}\right)\right)-1}, \\
& \left\{\begin{array}{c}
f_{H}=-\frac{1}{2 \pi^{2}} \int_{0}^{\infty} \frac{k^{4} \mathrm{~d} k}{\sqrt{k^{2}+m_{\pi}^{2}}\left(\mathrm{e}^{\frac{1}{T} \sqrt{k^{2}+m_{\pi}^{2}}}-1\right)} \\
f_{Q G P}=-\left(\frac{37}{90} \pi^{2} T^{4}+\mu^{2} T^{2}+\frac{\mu^{4}}{2 \pi^{2}}\right) \\
\quad-\frac{6}{\sqrt{2}}\left(\frac{m_{s} T}{\pi}\right)^{3 / 2} \mathrm{e}^{-m_{s} / T}\left(T+\frac{\mu^{2}}{2 ! T}+\frac{\mu^{4}}{4 ! T^{3}}\right)+B
\end{array}\right. \\
& \left\{\begin{array}{c}
s_{H}=\frac{1}{2 \pi^{2} T^{2}} \int_{0}^{\infty} \frac{k^{4} \mathrm{~d} k}{\mathrm{e}^{-\frac{1}{T} \sqrt{k^{2}+m_{\pi}^{2}}}\left(\mathrm{e}^{\frac{1}{T} \sqrt{k^{2}+m_{\pi}^{2}}}-1\right)^{2}} \\
\quad s_{Q G P}=\frac{74}{45} \pi^{2} T^{3}+2 \mu^{2} T+\frac{6}{\sqrt{2}}\left(\frac{m_{s} T}{\pi}\right)^{3 / 2} \mathrm{e}^{-m_{s} / T}(4) \\
\quad \times\left[\left(\frac{3}{2 T}+\frac{m_{s}}{T^{2}}\right)\left(T+\frac{\mu^{2}}{2 ! T^{2}}+\frac{\mu^{4}}{4 ! T^{4}}\right)+\left(1-\frac{\mu^{2}}{2 ! T^{2}}-\frac{3 \mu^{4}}{4 ! T^{4}}\right)\right]
\end{array}\right.
\end{aligned}
$$

*corresponding author; e-mail: lebba_ghenam@yahoo.fr 


$$
\left\{\begin{array}{c}
\varepsilon_{H}=-\frac{1}{2 \pi^{2}} \int_{0}^{\infty} \frac{k^{4} \mathrm{~d} k}{\sqrt{k^{2}+m_{\pi}^{2}}\left(\mathrm{e}^{\frac{1}{T} \sqrt{k^{2}+m_{\pi}^{2}}}-1\right)} \\
\quad+\frac{1}{2 \pi^{2} T} \int_{0}^{\infty} \frac{k^{4} \mathrm{~d} k}{\mathrm{e}^{-\frac{1}{T} \sqrt{k^{2}+m_{\pi}^{2}}}\left(\mathrm{e}^{\frac{1}{T} \sqrt{k^{2}+m_{\pi}^{2}}}-1\right)^{2}} \\
\varepsilon_{Q G P}=\frac{111}{90} \pi^{2} T^{4}+\mu^{2} T^{2}-\frac{\mu^{4}}{2 \pi^{2}}+\frac{6 \mathrm{e}^{-m_{s} / T}}{\sqrt{2}}\left(\frac{m_{s} T}{\pi}\right)^{3 / 2} \\
\quad \times\left[\left(\frac{1}{2}+\frac{m_{s}}{T}\right)\left(T+\frac{\mu^{2}}{2 ! T}+\frac{\mu^{4}}{4 ! T^{3}}\right)+\left(T-\frac{\mu^{2}}{2 ! T}-\frac{\mu^{4}}{4 ! T^{3}}\right)\right] \\
\quad+B
\end{array}\right.
$$

$k$ being the momentum and $m_{s}, m_{\pi}$ the strange quark and pion masses, respectively.

Figure 1 shows the three-dimensional plots of the order parameter (left), the energy density normalized by $T^{4}$ (middle) and the entropy density normalized by $T^{3}$ (right), vs. temperature and volume, at a vanishing chemical potential $\mu=0$. The first-order character of the transition is showed by the step-like rise of the order parameter, as well as the normalized energy and entropy densities, when approaching the thermodynamic limit, at a true transition temperature $T_{C}(\infty)$. This sharp discontinuity reflects the existence of a latent heat accompanying the phase transition. The quantities $\langle\varepsilon(T, V)\rangle / T^{4}$ and $\langle s(T, V)\rangle / T^{3}$ are traditionally interpreted as a measure of the number of effective degrees of freedom; the temperature increase causes then a melting of the constituent degrees of freedom frozen in the hadronic state, making the energy and entropy densities attain their plasma values. In small systems, the phase transition is rounded since the probability of presence of the QGP phase below $T_{C}$, and of the hadronic phase above $T_{C}$ are finite because of the considerable thermodynamical fluctuations. The transition region around the transition temperature is then broadened, acquiring a bigger width $\delta T(V)$, smaller is the volume.

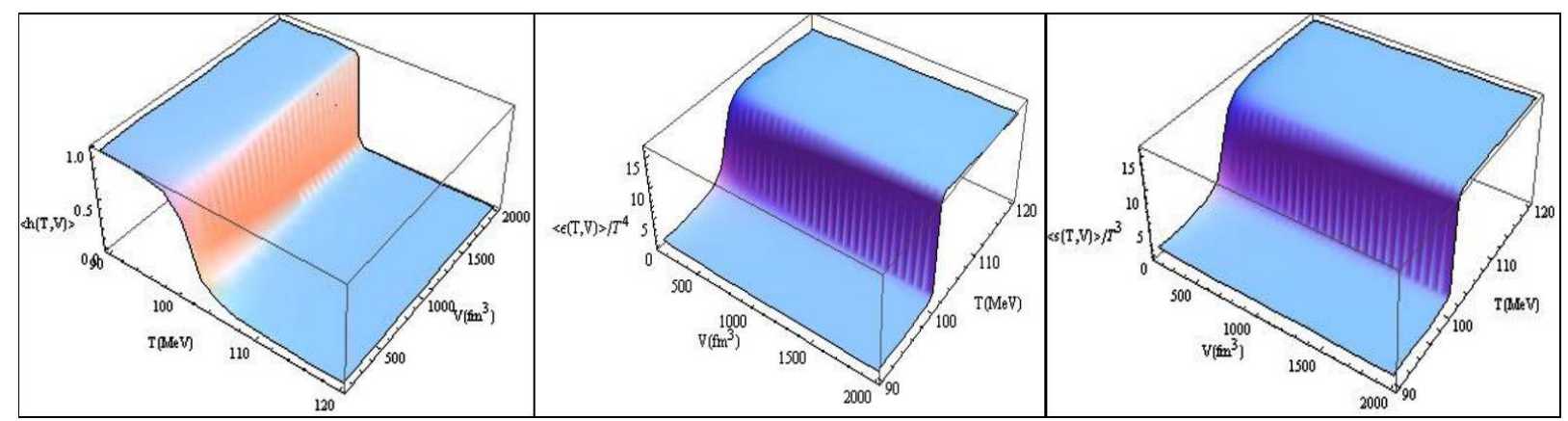

Fig. 1. Mean value of (left) the order parameter, (middle) the energy density normalized by $T^{4}$ and (right) the entropy density normalized by $T^{3}$ as functions of temperature $T$ and system size $V$, at $\mu=0$.

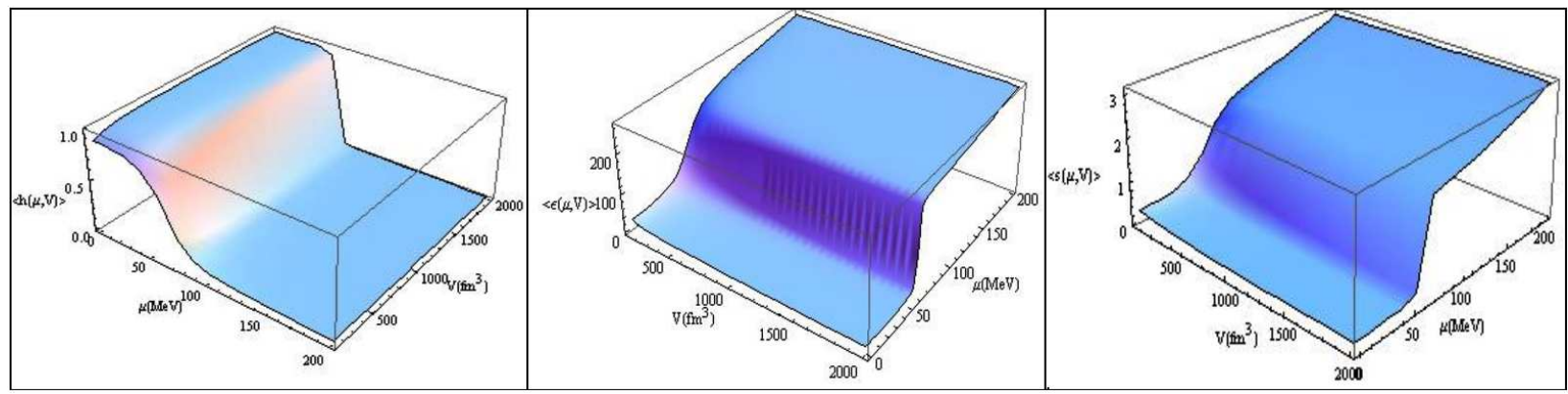

Fig. 2. Mean value of (left) the order parameter, (middle) the energy density and (right) the entropy density as functions of chemical potential $\mu$ and system size $V$, at the temperature $T=99 \mathrm{MeV}$.

Similarly, Fig. 2 shows the 3 -D variations of the order parameter, the energy and entropy densities with chemical potential and system volume, at a fixed temperature $T=99 \mathrm{MeV}$. The first-order character of the transition can, also in this case, clearly be seen from the sharp discontinuity of the three quantities at a transition chemical potential $\mu_{C}(\infty)$, at the large volume limit. In small systems, the transition is perfectly smooth over a broadened region of chemical potential of width $\delta \mu(V)$.
Figure 3 shows the $3-\mathrm{D}$ variations of the specific heat density $c(T, V)$ (left) and the susceptibility $\chi(T, V)$ (right) with temperature and system volume at $\mu=0$. These quantities are the derivatives of the energy density and the order parameter respectively, with respect to temperature. It can clearly be seen that the delta function singularity of both quantities occurring in the thermodynamical limit is smeared out, in a finite volume, into a finite peak of width $\delta T(V)$. For decreasing volume, 


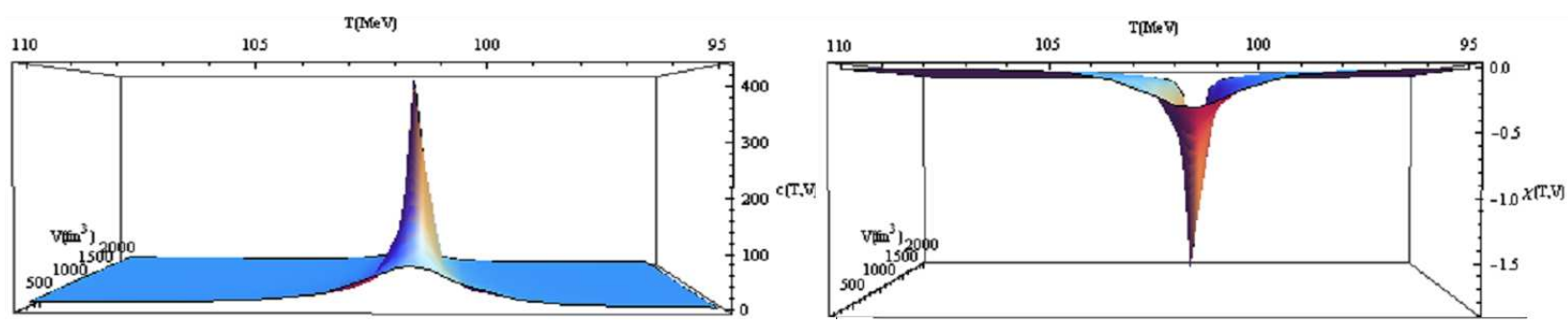

Fig. 3. The specific heat density (left) and the susceptibility (right) as function of temperature and volume at $\mu=0$.

the widths of the peaks get larger while their heights $c_{T}^{\max }(V)$ and $\chi_{T}^{\max }(V)$ decrease. Similar variations are also abtained for the specific heat density $c(\mu, V)$ and the susceptibility $\chi(\mu, V)$, which are now obtained respectively by derivating the energy density and the order parameter with respect to chemical potential.

Finite size effects in the case of the thermally driven as well as the density driven DPT, can then be summarized in the following points: the rounding of discontinuities, or equivalently the smearing of singularities at the level of the first derivatives, and the broadening of the transition region. To these effects can be associated three useful characteristic quantities for each of the two transitions, namely, the maxima of the peaks of both thermal susceptibility $\chi_{T}^{\max }(V)$ and specific heat density $c_{T}^{\max }(V)$, and the width of the transition region $\delta T(V)$ for the thermal DPT, and for the density driven DPT, the maxima of the peaks of both susceptibility $\chi_{\mu}^{\max }(V)$ and specific heat density $c_{\mu}^{\max }(V)$, and the width $\delta \mu(V)$ over which the transition is rounded off. Each of these quantities can be considered as an indicator of the order of the occurring transition, and is expected to exhibit a scaling behaviour in the form of a power law of the volume $V$, characterized by a scaling critical exponent. For a first-order thermal phase transition, the set of power laws is: $\chi_{T}^{\max }(V) \propto V^{\gamma} ; c_{T}^{\max }(V) \propto V^{\alpha} ; \delta T(V) \propto V^{-\theta}$, where the scaling exponents $\gamma, \alpha, \theta$ have been shown in the finite-size scaling theory to be all equal to unity [6].

The results of the present work with power law variations on the form: $\chi_{T}^{\max }(V) \propto V^{\gamma_{T}} c_{T}^{\max }(V) \propto$ $V^{\alpha_{T}} ; \delta T(V) \propto V^{-\theta_{T}}$ for the thermally driven DPT, and: $\chi_{\mu}^{\max }(V) \propto V^{\gamma_{\mu}} ; c_{\mu}^{\max }(V) \propto V^{\alpha_{\mu}} ; \delta \mu(V) \propto V^{-\theta_{\mu}}$ for the density driven DPT, give scaling exponents $\gamma_{T}, \alpha_{T}, \theta_{T}$ and $\gamma_{\mu}, \alpha_{\mu}, \theta_{\mu}$ all equal to unity, similarly to the results of $[2,3]$, where a finite size scaling analysis gave: $\gamma_{T}=\alpha_{T}=\theta_{T}=1$, signaling a first order thermal DPT.

\section{Conclusion}

In this work, we found that the behaviour of thermodynamic quantities characterizing the deconfinement phase transition in a finite volume, in the presence of massive particles, is similar to the case of massless particles [2, 3], signaling a first order character of the transition.

\section{References}

[1] C.N. Yang, T.D. Lee, Phys. Rev. 87, 404 (1952); T.D. Lee, C.N. Yang, Phys. Rev. 87, 410 (1952).

[2] M. Ladrem, A. Ait El Djoudi, Eur. Phys. J. C 44, 257 (2005).

[3] A. Ait El Djoudi, Ph.D. Thesis, Université Mouloud Mammeri, Tizi-Ouzou, Algeria, 2008.

[4] A. Ait El Djoudi, L. Ghenam, AIP Conf. Proc. 1444, 175 (2012).

[5] L. Ghenam, A. Ait El Djoudi, AIP Conf. Proc. 1444, 408 (2012).

[6] M.E. Fisher, A.N. Berker, Phys. Rev. B 26, 2507 (1982); M.S. Challa, D.P. Landau, K. Binder, Phys. Rev. B 34, 1841 (1986). 